\title{
Spinal Epidural Lipomatosis Associated with Intrathecal Flow Voids: Demonstration of Engorged Veins Using Flat Panel Catheter Angiotomography
}

\author{
Nishtha Yadav ${ }^{1}$ Ketan Hedaoo ${ }^{2}$ Ambuj Kumar ${ }^{2}$ \\ ${ }^{1}$ Department of Neuroradiology, School of Excellence in \\ Neurosurgery, Super Speciality Hospital, Netaji Subhash Chandra \\ Bose Medical College, Jabalpur, Madhya Pradesh, India \\ 2 Department of Neurosurgery, Super Speciality Hospital, Netaji \\ Subhash Chandra Bose Medical College, Jabalpur, Madhya Pradesh, \\ India
}

\author{
Address for correspondence Nishtha Yadav, DM, Department of \\ Neuroradiology, School of Excellence in Neurosurgery, Super \\ Speciality Hospital, Netaji Subhash Chandra Bose Medical College, \\ Jabalpur, Madhya Pradesh 482003, India \\ (e-mail: nishthayadav@yahoo.com).
}

J Neurosci Rural Pract 2022;13:137-140.

\author{
Abstract \\ Keywords \\ - spinal epidural \\ lipomatosis \\ - flow voids \\ - flat panel catheter \\ angiotomography \\ - spinal angiography
}

We present a case of a 54-year-old male with spinal epidural lipomatosis who had associated flow voids on magnetic resonance imaging with dilated intrathecal vessels. During spinal angiogram, 20s DynaCT (flat panel catheter angiotomography) was utilized to demonstrate the intrathecal engorged veins. Venous engorgement of epidural venous plexus has been previously described in epidural lipomatosis; however, dilated intrathecal perimedullary veins have not been demonstrated by imaging. We have described the utility of flat panel catheter angiotomography in understanding venous disorders in such patients.

\section{Introduction}

Spinal epidural lipomatosis is a rare and complex disorder that can present with progressive neurological deficits and is caused by hypertrophy of adipose tissue in spinal epidural space. We present a rare case of spinal epidural lipomatosis associated with flow voids on magnetic resonance imaging (MRI). In our patient, the flow voids were due to intrathecal perimedullary vein engorgement, which was demonstrated using flat panel catheter angiotomography (FPCA). Although flow voids due to engorged veins in spinal epidural lipomatosis are rarely found, high degree of suspicion of venous pathology in such cases (rather than a spinal vascular malformation) should be considered especially in a setting of normal spinal angiography and FPCA should be utilized in demonstration of the same.

\section{Case Report}

A 54-year-old male presented with history of gradually progressive weakness for past 2 years. At the time of presentation, he was using cane for walking. There was no history of trauma, fever, drug intake, or infections. No history of bowel/bladder incontinence was noted. Physical examination revealed body mass index $22 \mathrm{~kg} / \mathrm{m}^{2}$ (weight $60 \mathrm{~kg}$, height $165 \mathrm{~cm}$ ) without any signs of endocrinopathies/Cushing's syndrome. Neurological examination revealed increased tone in bilateral lower limbs with preserved muscle bulk. Power in bilateral lower limbs was $4 / 5$ (Medical Research Council grading) with brisk knee and ankle jerks. Bilateral planters were extensor. There was no sensory loss. There was no spinal deformity or tenderness. MRI was done which revealed T1, T2 hyperintense lesion published online December 30, 2021
DOI https://doi.org/

10.1055/s-0041-1736510. ISSN $0976-3147$. (c) 2021. Association for Helping Neurosurgical Sick People. All rights reserved.

This is an open access article published by Thieme under the terms of the Creative Commons Attribution-NonDerivative-NonCommercial-License, permitting copying and reproduction so long as the original work is given appropriate credit. Contents may not be used for commercial purposes, or adapted, remixed, transformed or built upon. (https://creativecommons.org/ licenses/by-nc-nd/4.0/)

Thieme Medical and Scientific Publishers Pvt. Ltd., A-12, 2nd Floor, Sector 2, Noida-201301 UP, India 


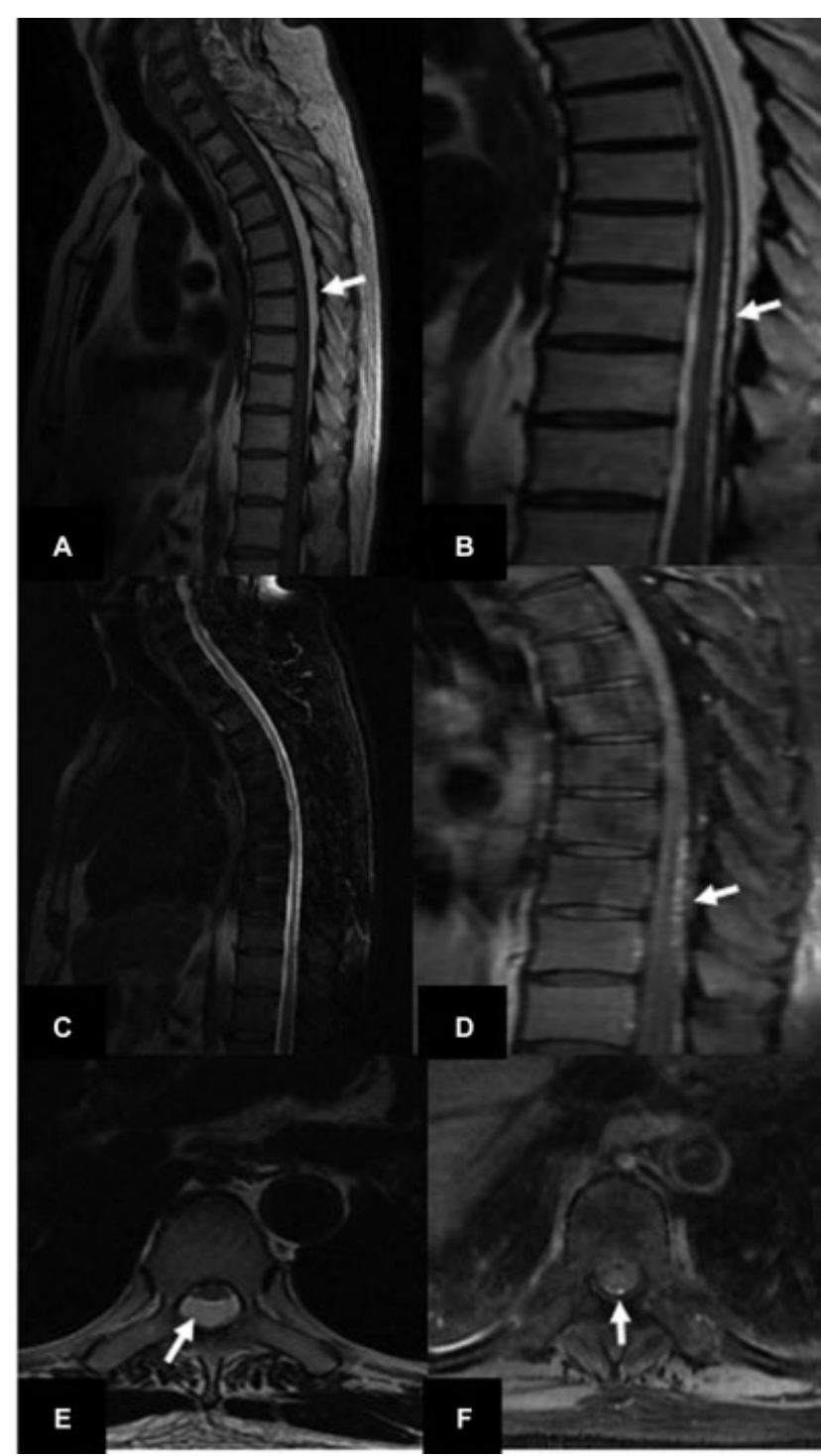

Fig. 1 (A) T1-weighted (T1W) sagittal image shows T1 hyperintense lesion extending from D2 to D9 level in posterior epidural space (white arrow). (B) T2W sagittal image shows the lesion is hyperintense on T2 and is causing anterior displacement of dura and anterior displacement of cord with presence of intrathecal flow voids (white arrow). (C) T2W fat saturated image shows suppression of signal in posterior epidural space (suggestive of fat signal). (D) Postcontrast T1W sagittal image shows serpiginous enhancement posterior to cord from D9 to D11 levels (white arrow). (E) T2W axial image shows T2 hyperintense posterior epidural lesion causing anterior displacement of cord with cord compression (white arrow). (F) Postcontrast T1W fat saturated axial image shows enhancing vessel posterior to cord at D10 level (white arrow).

in posterior epidural space extending from D2 to D9 levels, causing spinal canal compromise and anterior displacement of cord ( - Fig. 1). The lesion showed hypointense signal on fat saturated images and no enhancement was noted. No cord signal changes were noted. Intrathecal flow voids were noted from D9 to D11 levels, with presence of serpiginous enhancement posterior to cord at same location ( - Fig. 1). Diagnosis of thoracic epidural lipomatosis was made with spinal canal stenosis and myelopathy. However, due to presence of myelopathy along with intrathecal flow voids, a spinal angiogram

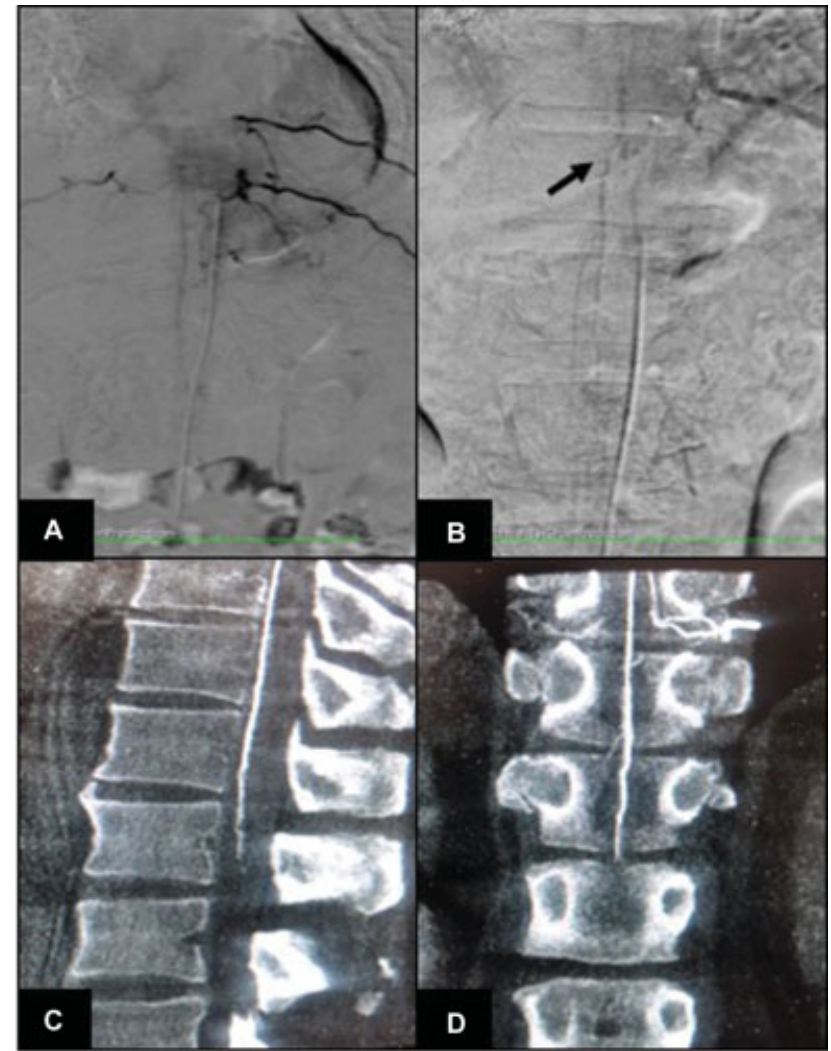

Fig. 2 (A) Spinal angiography digital subtraction angiography image in arterial phase shows origin of artery of Adamkiewicz from left D10 intercostal artery; no opacification of fistula/early veins is noted. (B) Venous phase spinal angiography image (at 15 seconds) shows visualization of engorged spinal perimedullary veins (black arrow). (C) Sagittal and (D) coronal reconstruction of $5 \mathrm{~s}$ DynaCT (flat panel catheter angiotomography) image show opacification of anterior spinal artery (Artery of Adamkiewicz), without visualization of perimedullary veins.

was planned to rule out a coexisting spinal vascular malformation.

Spinal angiogram did not reveal evidence of spinal vascular malformation. Injection of left D10 intercostal artery showed opacification of artery of Adamkiewicz. Delayed acquisition revealed venous phase at 15 seconds with visualization of engorged veins at the same level as noted on MRI (D9-D11) (-Fig. 2). Initial FPCA was done after selective injection of left D10 intercostal artery using a 5-second acquisition (DynaCT) on Artis zee, (Siemens, Erlangen, Germany). Continuous hand injection of $10 \mathrm{~mL}$ of $50 \%$ diluted iodinated contrast agent $(300 \mathrm{mgI} / \mathrm{mL})$ was done using $20 \mathrm{cc}$ syringe while acquiring 5 s DynaCT. Data was reconstructed using high-resolution matrices $(0.1 \mathrm{~mm}$ voxel size). There was opacification of anterior spinal artery, with no opacification of early draining veins/fistula (-Fig. 2). After 5 minutes, another FPCA was done after similar selective injection of left D10 intercostal artery using a 20-second acquisition (DynaCT, Siemens). The reconstructed image evaluation revealed presence of engorged anterior median spinal vein, posterior median spinal vein, extrinsic surface anastomosis between anterior and posterior median vein, and few other dilated perimedullary veins (-Fig. 3). The 


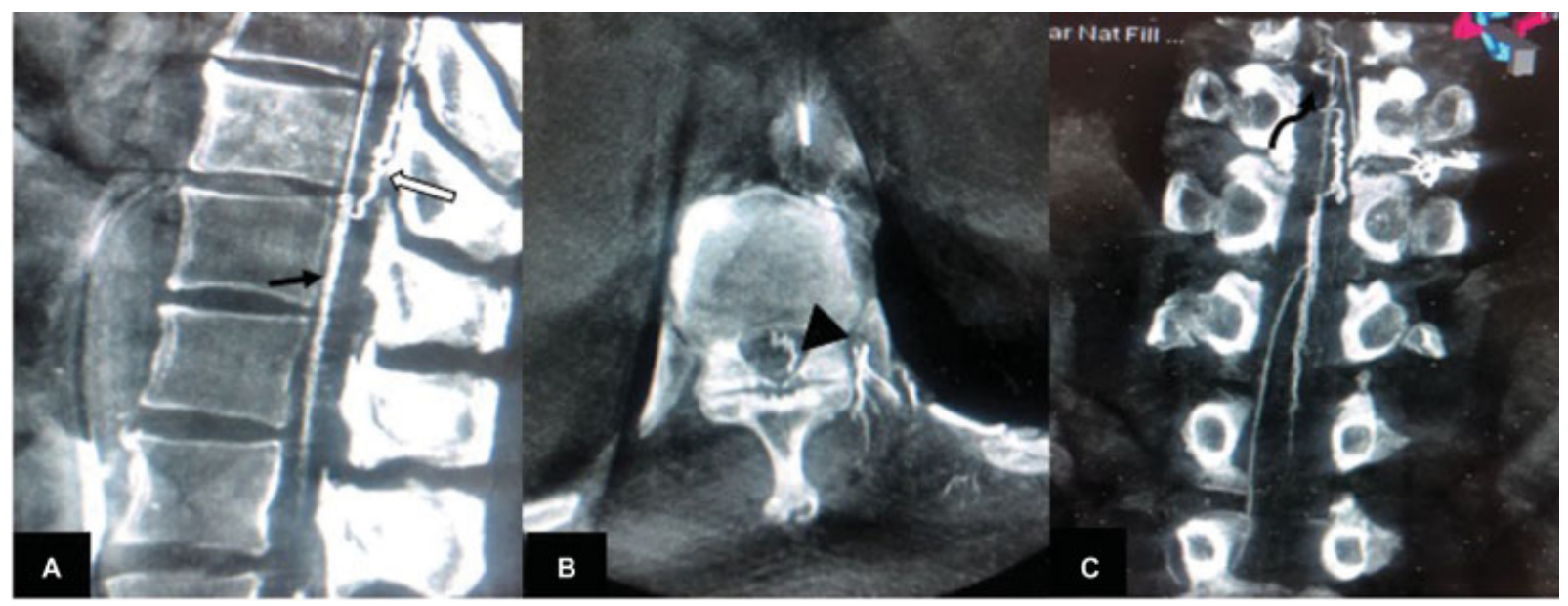

Fig. 3 (A) Sagittal, (B) axial, and (C) coronal reconstruction of 20s DynaCT (flat panel catheter angiotomography) show visualization of anterior median spinal vein (black arrow in $[A]$ ), posterior median spinal vein (white arrow in $[A]$ ), extrinsic surface anastomosis between anterior and posterior median vein (arrowhead in [B]), and engorged perimedullary veins (curved arrow in [C]).

engorged perimedullary veins demonstrated on FPCA were considered the cause of flow voids on MRI and spinal vascular malformation was ruled out. The patient underwent surgical excision of epidural lipomatosis and had improvement in weakness postoperatively.

\section{Discussion}

Spinal epidural lipomatosis can present with back pain, limb weakness, sensory changes, radicular pain, or rarely bowel/bladder incontinence. The neurological symptoms are dependent on the degree and level of canal compromise (cervical/thoracic/lumbar leading to cord/conus/cauda equine compression). ${ }^{1}$ Myelopathy is attributed to direct effects of mechanical compression due to spinal canal stenosis as well as indirectly due to compression of the epidural blood vessels with subsequent venous engorgement. ${ }^{2-4}$ Intraoperative epidural venous engorgement has been noted during excision of epidural lipomatosis. ${ }^{2}$ Engorgement of epidural venous with epidural lipomatosis has been described by Park et al on MRI. ${ }^{5}$ However, dilatation of intrathecal perimedullary veins has rarely been described previously.

Presence of myelopathy along with flow voids on MRI requires further imaging in form of spinal angiogram to rule out spinal vascular malformation. In a study by Alhilali et al, they aimed to determine prevalence of spinal dural arteriovenous fistulae (SDAVF) in patients presenting with prominent vascular flow voids on imaging without other imaging findings suggestive of SDAVF. ${ }^{6}$ They studied 18 patients with flow voids on MRI, without any other imaging evidence of spinal vascular malformation (such as cord signal change/ venous infarct), and found only $17 \%$ patients had SDAVF on angiography, and all of them had myelopathy symptoms. They concluded that prominent flow voids without other imaging findings suggestive of SDAVF are poorly predictive of the presence of a SDAVF, unless myelopathy is present clinically. ${ }^{6}$ They also noted a negative spinal angiogram in one of their patients who had associated epidural lipomatosis, similar to our patient. ${ }^{6}$ In our case, we were further able to demonstrate the engorged veins in venous phase on spinal angiogram using FPCA.

Demonstration of venous phase in spinal digital subtraction angiography (DSA) is difficult because it requires the patient to hold the breath for 15 to 20 seconds. Additionally, bowel peristalsis and poor bowel preparation also make it difficult to evaluate venous phase on spinal angiogram. Even though it is generally considered that demonstration of venous phase (within 18 seconds) usually indicates absence of spinal vascular malformation ${ }^{7}$ this is not always true. In a study by Eckart Sorte et al, they concluded that the mere observation of a normal venous phase cannot be used to exclude the presence of a vascular malformation or justify interrupting a diagnostic spinal angiogram. ${ }^{8}$ However, demonstration of venous phase/perimedullary veins is very useful and improves the understanding of venous system anatomy and hemodynamics.

Previous studies have evaluated the utility of FPCA as an adjunct to spinal angiography and have found it to be particularly valuable in detection of spinal venous anomalies. ${ }^{9-11}$ FPCA has many advantages for the demonstration of spinal venous phase. FPCA provides high-resolution images of the spinal vasculature, particularly, of the spinal veins, in any desired plane. ${ }^{10}$ Moreover, there is no need for breath hold because breathing and bowel peristalsis artifacts do not affect the quality of reconstructed images in FPCA. ${ }^{10}$ The rich information provided by FPCA helps in the treatment planning for vascular malformations. ${ }^{12}$ Additionally, FPCA can transform a "negative spinal angiogram" into a positive angiographic diagnosis, thus eliminating the need of repeat studies/additional investigations for such patients that are usually performed due to the fear of an overlooked lesion after a negative spinal angiogram. ${ }^{10}$ Our case exemplifies this situation. While presence of myelopathy along with intrathecal flow voids on MRI had raised the suspicion of a 
vascular malformation in our patient, the spinal DSA was normal. However, the findings on FPCA showing engorged veins are correlated with the abnormalities on MRI (flow voids and serpiginous enhancement) along the dorsal surface of the spinal cord in the same location. Epidural lipomatosis has been known to cause compression of epidural veins with venous engorgement. Compacted epidural venous plexus may lead to transmission of increased pressure to perimedullary veins with resultant intrathecal venous engorgement. This case further demonstrates the usefulness of FPCA technique in improving spinal venous imaging. FPCA can help in better understanding of poorly understood conditions involving the spinal venous system and in future can possibly demonstrate venous disorders that are not as yet appreciated. $^{10}$

\section{Conclusion}

We report a case of spinal epidural lipomatosis with associated intrathecal flow voids. Venous engorgement of epidural venous plexus has been previously described in epidural lipomatosis; however, dilated intrathecal perimedullary veins have not been demonstrated by imaging. We have described the utility of FPCA in understanding venous disorders in such patients.

\section{Ethical Approval}

The study has been approved by the appropriate ethics committee and has therefore been performed in accordance with the ethical standards laid down in the 1964 Declaration of Helsinki and its later amendments.

\author{
Funding \\ None. \\ Conflict of Interest \\ None declared.
}

\section{References}

1 Fassett DR, Schmidt MH. Spinal epidural lipomatosis: a review of its causes and recommendations for treatment. Neurosurg Focus 2004;16(04):E11

2 Akhaddar A, Ennouali H, Gazzaz M, Naama O, Elmostarchid B, Boucetta M. Idiopathic spinal epidural lipomatosis without obesity: a case with relapsing and remitting course. Spinal Cord 2008; 46(03):243-244

3 Rajput D, Srivastava AK, Kumar R. Spinal epidural lipomatosis: an unusual cause of relapsing and remitting paraparesis. J Pediatr Neurosci 2010;5(02):150-152

4 Papastefan ST, Bhimani AD, Denyer S, et al. Management of idiopathic spinal epidural lipomatosis: a case report and review of the literature. Childs Nerv Syst 2018;34(04):757-763

5 Park SK, Lee IS, Song YS, Moon JI, Song JW, Kang H. Dilatation of the spinal epidural venous plexus in patients with prominent epidural fat. Br J Radiol 2016;89(1063):20160064. Doi: 10.1259/ bjr.20160064

6 Alhilali LM, Reynolds AR, Fakhran S. Value of prominent flow voids without cord edema in the detection of spinal arteriovenous fistulae. PLoS One 2014;9(06):e99004. Doi: 10.1371/journal.pone.0099004

7 Willinsky R, Lasjaunias P, Terbrugge K, Hurth M. Angiography in the investigation of spinal dural arteriovenous fistula. A protocol with application of the venous phase. Neuroradiology 1990;32 (02):114-116

8 Eckart Sorte D, Obrzut M, Wyse E, Gailloud P. Normal venous phase documented during angiography in patients with spinal vascular malformations: incidence and clinical implications. AJNR Am J Neuroradiol 2016;37(03):565-571

9 Gregg L, Gailloud P. Transmedullary venous anastomoses: anatomy and angiographic visualization using flat panel catheter angiotomography. AJNR Am J Neuroradiol 2015;36(07):1381-1388

10 Chen J, Ethiati T, Gailloud P. Flat panel catheter angiotomography of the spinal venous system: an enhanced venous phase for spinal digital subtraction angiography. AJNR Am J Neuroradiol 2012;33 (10):1875-1881

11 Pearl MS, Chen JX, Gregg L, et al. Angiographic detection and characterization of "cryptic venous anomalies" associated with spinal cord cavernous malformations using flat-panel catheter angiotomography. Neurosurgery 2012;71(1, Suppl Operative):125-132

12 Aadland TD, Thielen KR, Kaufmann TJ, et al. 3D C-arm conebeam $\mathrm{CT}$ angiography as an adjunct in the precise anatomic characterization of spinal dural arteriovenous fistulas. AJNR Am J Neuroradiol 2010;31(03):476-480 